\title{
Surgical-interventional hybrid orchestra consisting of Potts shunt, transcatheter tricuspid valve repair by Edwards-valve in a 26-year-old patient with pulmonary hypertension and right ventricular failure
}

\author{
Sabine Recla ${ }^{1}$, Blanka Steinbrenner ${ }^{1}$, Jennifer Schreier ${ }^{2}$, Stephan Fichtlscherer ${ }^{2}$, Dorle Schmidt ${ }^{1}$, \\ Christian Apitz $^{1}$, Matthias Müller ${ }^{1}$, Juergen Bauer ${ }^{1}$, Hakan Akintuerk ${ }^{1}$, Dietmar Schranz ${ }^{*}$ \\ ${ }^{1}$ Hessiches Kinderherzzentrum, Universitätskliniken Giessen, Frankfurt, Germany \\ ${ }^{2}$ Medizinische Klinik III, Kardiologie Universitätsklinikum, Frankfurt, Germany \\ Email: ${ }^{\text {dietmar.schranz@paediat.med.uni-giessen.de }}$ \\ Received 30 June 2013; revised 5 August 2013; accepted 21 August 2013 \\ Copyright (c) 2013 Sabine Recla et al. This is an open access article distributed under the Creative Commons Attribution License, \\ which permits unrestricted use, distribution, and reproduction in any medium, provided the original work is properly cited.
}

\begin{abstract}
Reported here is a 26-year-old patient with corrected congenital interrupted aortic arch admitted with massive hemoptysis and severe suprasystemic pulmonary hypertension provided him for heart-lung transplantation. Initial closure of a small aorto-pulmonary collateral remained ineffective. Persistent lifethreatening hemoptysis led to the decision for a modified Potts shunt with the aim of decompressing the right ventricle and avoiding pulmonary-hypertensive crisis. The hemoptysis did not recur. However, the patient's oxygen transport condition deteriorated and necessitated an orchestra of interventional-surgical approaches from re-coarctation stenting, Potts shunt flow reducing to surgical and transcatheter tricuspid valve repair with transient ECMO before the patient could be discharged home.
\end{abstract}

Keywords: Pulmonary Hypertension; Potts Shunt; Edwards Tricuspid Valve; Hybrid-Procedure

\section{INTRODUCTION}

Pulmonary hypertension ( $\mathrm{PH})$ with hemoptysis and suprasystemic pressures is associated with poor prognosis [1-3]. Decrease of cardiac output is not only related to impaired right ventricular (RV) function, but also a consequence of impaired LV filling due to right-to-left ventricular interaction [4]. As a novel approach for palli-

\footnotetext{
${ }^{*}$ Corresponding author.
}

ating children with suprasystemic idiopathic pulmonary arterial hypertension (PAH), surgical Potts shunt has been reported as an alternative treatment to lung transplantation $[5,6]$.

\section{CASE REPORT}

A 26-year-old young man (body weight $52 \mathrm{~kg}$ ) was emergently referred with massive hemoptysis. He was known to have suprasystemic pre-capillary PH secondary to a complex congenital heart malformation (IAA-Type B). Reconstruction of the aortic arch by a modified Waldhausen technique combined with a pulmonary artery banding was performed in infancy. VSD closure and pulmonary artery de-banding followed at the age of 2 years. 15 years later aortic arch was re-reconstructed by PTFE-patch enlargement. He was known to have precapillary pulmonary hypertension, which had been treated by a calcium-antagonist, with the later addition of sildenafil and bosentan.

At admission life-threatening hemoptysis was associated with right ventricular dilatation $\left(143 \mathrm{ml} / \mathrm{m}^{2}, \mathrm{MRI}\right)$ severe tricuspid valve insufficiency and significant cyanosis necessitating continuous supplemental oxygen therapy. Heart catheterization was performed but only one small collateral vessel could be detected and closed by coils, but hemoptysis persisted. The suprasystemic pulmonary arterial pressure (PAP) was confirmed with PAP to systemic artery pressure (SAP) ratio of 1.2 and 1.5 after contrast medium injection which was used to delineate the relationship between the left pulmonary 
artery (LPA) and descending aorta (Figure 1). Nitric oxide test-inhalation (20 ppm) did not show any significant effect on the PAH (PAP 102/63/76 mmHg; corresponding PCWP of $16 \mathrm{mmHg}$, systemic arterial pressure (SAP) in the ascending aorta of 95/61/73 mmHg, descending aorta (DAO) of 84/58/66 mmHG). After detailed discussion between the heart team, the patient and his family led to the decision for a surgical Potts shunt rather then listing the patient for combined lung- or heartlung transplantation. Based on the data from France [6], a $13 \mathrm{~mm}$ PTFE-graft tube was surgically connected from the left-PA to the DAO (Figure 2). The left-intercostal surgical approach was technically uneventful. Hemoptysis was not observed again. However, the combination of a post-operative left-lower lobe atelectasis, newly created right-to-left shunt through the PTFE-tube and extreme rarefied pulmonary vascular bed resulted in an extreme lower body oxygen saturation of $28 \%$ distal to the Potts shunt with an associated inferior caval vein (IVC) oxygen saturation of $13 \%$, but without an increased systemic lactate level. The superior caval vein oxygen saturation declined to $31 \%$ along with to a low left ventricular cardiac output. The MRI blood-flow measurements in the pulmonary artery demonstrated $61 \mathrm{ml} /$ beat, $25 \mathrm{ml}$ in the ascending, and $12 \mathrm{ml}$ in the descending aorta pre-, and $45 \mathrm{ml} /$ beat post-Potts shunt, respectively. A reduced left ventricular preload with consecutive low left ventricular cardiac output was considered as a co-factor for the extreme lower body desaturation. Therefore, the slightly re-

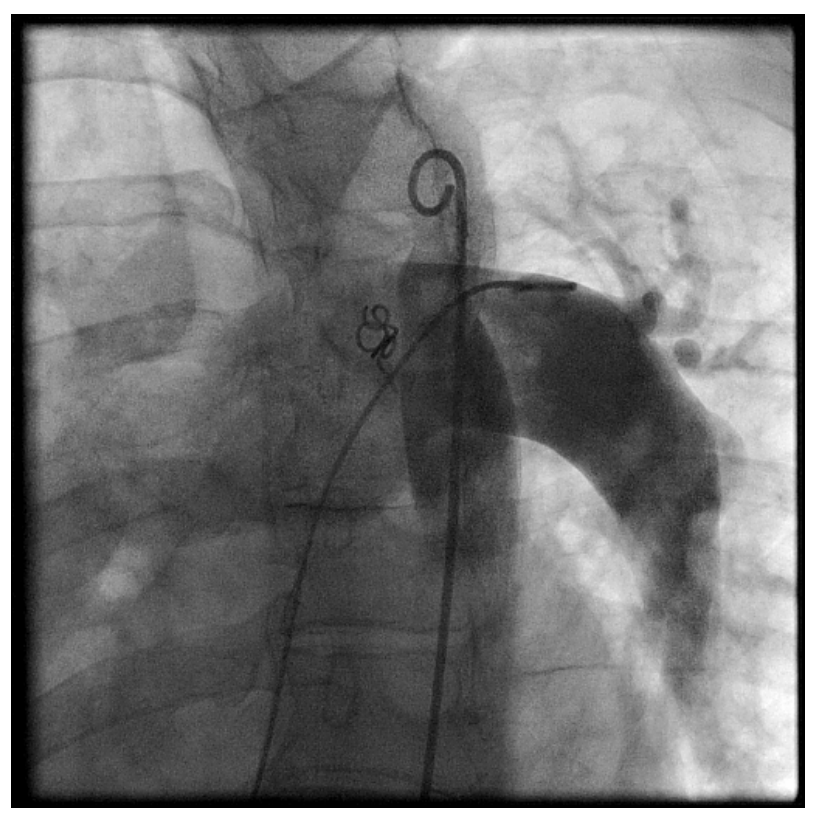

Figure 1. Demonstrates simultaneous injection of contrast medium in the descending aorta (DAO) and left pulmonary artery (LPA) to evaluate the morphology prior to Potts shunt surgery. Measured LPA diameter was $24 \mathrm{~mm}$, and DAO $16 \mathrm{~mm}$, respectively.

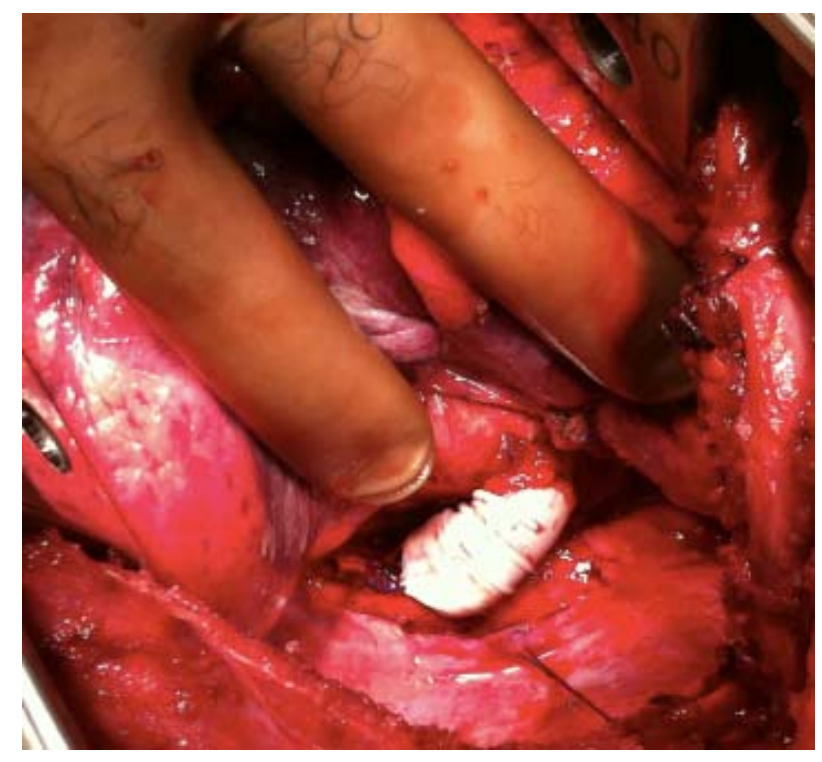

Figure 2. Depicts the surgical performed PTFE-graft after connecting the LPA to the DAO.

coarctated descending aortic arch was stented by an Andra $39 \mathrm{~mm}$ XL stent mounted on a $20 \times 45 \mathrm{~mm}$ Balloonin-Balloon (PFM). However, afterload reduction by diminishing the gradient across the aortic arch less than 5 mmHg improved only slightly the oxygen transport parameters. Therefore, we decided for flow reducetion through the Potts shunt. A premounted $29 \times 14 \mathrm{~mm}$ Atrium graft stent (Advanta V12) advanced from a venous access was implanted in a diabolo shape by a slow flared balloon inflation from out-side in until a narrowed part of $7 \mathrm{~mm}$ in the middle of the graft-stent was achieved. Thereafter measurements of oxygen transport parameters improved. The additional trial to create an interatrial communication for improving the left ventricular preload by atrioseptostomy using the Brockenbrough technique and gradual static balloon dilatation of atrial septum resulted in ischemic ECG changes and hemodynamic instability. Immediate device closure (Amplatzer ASD-12 mm, AGA) was performed to treat the hypoxemic reaction of the upper body part in particular of the coronary perfusion. The patient recovered slowly but he remained bed-dependent as a NYHA functional class of IV. Despite some clinical improvement the tricuspid regurgitation increased progressively. Again, surgical tricuspid valve reconstruction was considered as an alternative to listing for tricuspid repair together with lung transplantation. Surprisingly, the surgical approach was well tolerated despite the need for an open-heart surgery, However, the reconstructed tricuspid valve utilizing a Carpentier-Edwards $30 \mathrm{~mm}$ band, remained sufficient for just 2 - 3 weeks. Due to progressive grad III regurgitation again, an off label use of a transcatheter 29 mm Edwards-Sapien Heart valve was considered to 
avoiding an additional open-heart surgery. Transcatheter valve implantation was performed under general anesthesia with fluoroscopic and 3D-TEE guidance. An $18 \mathrm{~F}$ Cook sheath was placed in femoral vein, and after sizing of the Carpentier-band, a $39 \mathrm{~mm}$ Andra XXL stent mounted on $30 \times 60 \mathrm{~mm}$ balloon catheter (Balt) was positioned in the tricuspid-annulus and slight diabolo shape created by expanding to $30 \mathrm{~mm}$ at both stent-ends followed by using a $30 \times 40 \mathrm{~mm}$ nucleus balloon (PFM). The already prepared Edwards-valve was immediately implanted within the previous Andra-stent and in the metal band-marked tricuspid valve annulus. However, the abrupt afterload increase after placement of the competent Edwards-valve caused the right ventricular function to deteriorate. Balloon dilatation of the Atrium stent within the Potts shunt to almost $9 \mathrm{~mm}$ diameter and Increasing dosages of catecholamines in addition to the baseline inotropic therapy with milrinone and levosimendan failed to stabilize his circulation. Therefore, the patient was put on ECMO (Extra-Corporal Membrane Oxygenation) by utilizing the percutaneous femoral vein (22 Fr sheath) and artery (16 Fr sheath) access. Bi-ventricular and in particular right ventricular function recovered over 5-days of ECMO therapy, and 7 days ventilatory support. The "hybrid orchestra" of interventional-surgical procedures is summarized on Figure 3.

Three weeks later, after a hospitalization for a total of 4.5 months the patient was discharged home, but still with a functional class NYHA III prior to further rehabilitation.

\section{COMMENT}

Patients with congenital heart disease dispose to PAH, particularly if the cardiac defect is left unrepaired, but even after corrective surgery due to inappropriate timing or ineffective initial palliation [7]. Comparing patients who could be transplanted and who died waiting had a significantly higher incidence of supra-systemic right heart pressures and hemoptysis [8,9], various rescue treatments have been proposed. Atrial septostomy is currently recommended for bridging to lung transplantation [10]. Recently, a Potts shunt has been described in patients with end-stage IPAH with suprasystemic PA pressures who are refractory to medical treatment $[5,6]$. This pulmonary-to-systemic connection changes cardiac pathophysiology into that of Eisenmenger's physiology. At our institution, Potts shunt combined with balloon atrial septostomy was successfully performed in one other patient with out of proportion post-capillary PAH associated with a borderline LV-morphology [11].

However, we underestimated the effect of the Potts shunt on the oxygen transport parameters, and in particular the negative effect on the left ventricular preload.

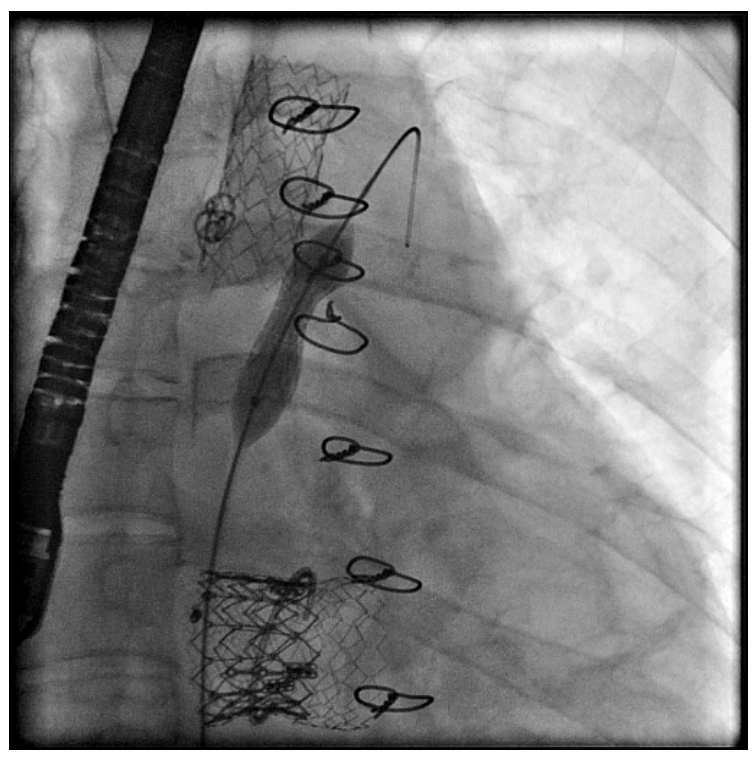

Figure 3. Depicted are all utilized devices for several transcatheter interventions. In neighborhood of the Andrastent placed in the aortic re-coarctation, the Jackson-coil in a aortopulmonary collateral is shown; the ensemble of the Andra- and Edwards-Sapien $29 \mathrm{~mm}$ valve positioned within the Carpentier banded tricuspid valve slightly covert the $12 \mathrm{~mm}$ Amplatzer ASD-occluder (see text); additionally a $10 \mathrm{~mm}$ inflated high pressure balloon is shown during slight re-dilatation of the Atrium V12-graft stent which was previously placed within the Potts Shunt; the dilatation of the graft-stent within the LPA-DAO-shunt to $10 \mathrm{~mm}$ was performed with the goal to reduce the afterload of the failing right ventricle after sufficient Edwards-valve placement.

Therefore, consecutive interventional-surgical measures consisting of aortic coarctation stenting, Potts shunt flow reduction; atrioseptostomy and immediate device closure, surgical and finally transcatheter tricuspid valve repair latest with the need for transient ECMO became necessary. However, the right ventricle recovered with balancing of the right-left ventricular interacttion, which allowed patients to discharge home.

This case highlights the utility of a surgical-intervenetional hybrid strategy in the treatment of heart failure with severe $\mathrm{PAH}$, considered at the highest risk for adverse surgical or even transplant outcomes. The shortterm efficacy of the appropriate trimmed Potts shunt and the $29 \mathrm{~mm}$-Edwards-valve in the tricuspid position in severe $\mathrm{PAH}$ is reassuring.

\section{REFERENCES}

[1] Benza, R.L., Miller, D.P., Barst, R.J., et al. (2012) An evaluation of long-term survival from time of diagnosis in pulmonary arterial hypertension. Chest, 142, 448-456. http://dx.doi.org/10.1378/chest.11-1460

[2] Raymond, R.J., Hinderliter, A.L., Willis, P.W., et al. 
(2002) Echocardiographic predictors of adverse outcomes in primary pulmonary hypertension. Journal of the American College of Cardiology, 39, 1214-1219. http://dx.doi.org/10.1016/S0735-1097(02)01744-8

[3] Zylkowska, J., Kurzyna, M., Pietura, R., et al. (2011) Recurrent hemoptysis: An emerging life-threatening complication in idiophathic pulmonary arterial hypertension. Chest, 139, 690-693. http://dx.doi.org/10.1378/chest.10-1134

[4] Gan, C.T., Lankhaar, J.W., Marcus, J.T., et al. (2006) Impaired left ventricular filling due to right-to-left ventricular interaction in patients with pulmonary arterial hypertension. American Journal of Physiology-Heart and Circulatory Physiology, 290, H1528-H1533.

[5] Blanc, J., Vouhe, P. and Bonnet, D. (2004) Potts shunt in patients with pulmonary hypertension. The New England Journal of Medicine, 350, 623. http://dx.doi.org/10.1056/NEJM200402053500623

[6] Baruteau, A.E., Serraf, A., Lévy, M., et al. (2012) Potts shunt in children with idiopathic pulmonary arterial hypertension: Long-term results. The Annals of Thoracic Surgery, 94, 817-824.

http://dx.doi.org/10.1016/j.athoracsur.2012.03.099

[7] Beghetti, M. and Tissot, C. (2010) Pulmonary hypertension in congenital shunts. Revista Española de Cardiología, 63, 1179-1193.
http://dx.doi.org/10.1016/S0300-8932(10)70250-5

[8] Cantu, J., Wang, D. and Safdar, Z. (2012) Clinical implications of haemoptysis in patients with pulmonary arterial hypertension. International Journal of Clinical Practice, 177, 5-12. http://dx.doi.org/10.1111/ijcp.12004

[9] Goldstein, B.S., Sweet, S.C., Mao, J., Huddleston, C.B. and Grady, R.M. (2011) Lung transplantation in children with idiopathic pulmonary arterial hypertension: An 18year experience. The Journal of Heart and Lung Transplantation, 30, 1148-1152. http://dx.doi.org/10.1016/j.healun.2011.04.009

[10] Micheletti, A., Hislop, A.A., Lammers, A., et al. (2006) Role of atrial septostomy in the treatment of children with pulmonary arterial hypertension. Heart, 92, 969-972. http://dx.doi.org/10.1136/hrt.2005.077669

[11] Latus, H., Apitz, C., Schmidt, D., Jux, C., Müller, M., Bauer, J., Akintuerk, H., Schneider, M. and Schranz, D. (2013) Potts shunt and atrioseptostomy in pulmonary hypertension due to left ventricular disease. The Annals of Thoracic Surgery, 96, 317-319.

http://dx.doi.org/10.1016/j.athoracsur.2012.10.069 